\title{
Применение газовой хромато-масс-спектрометрии для диагностики заболеваний надпочечников (обзор литературы)
}

Е. В. Малеваная К. Х.Н., Е. Г. Стрельникова К. Х.Н., Северо-Западный государственный медицинский университет им. И. И. Мечникова, Санкт-Петербург Н. Н. Зыбина д. б. н., Всероссийский центр экстренной и радиационной медицины имени А. М. Никифорова, Санкт-Петербург e.malevanaia@gmail.com
УДК 543.51; 543.54

Статья получена 25.10.2019 Принята к публикации 05.11.2019

Возможность определения конечных и промежуточных продуктов стероидогенеза делает хроматографические методы анализа, особенно хромато-масс-спектрометрию, наиболее ценными в комплексной диагностике заболеваний гипофизарно-адреналовой системы. В обзоре описаны различные стратегии пробоподготовки биологических образцов перед проведением анализа методом газовой хромато-масс-спектрометрии (ГХ-МС). Рассмотрены стадии ферментативного гидролиза, экстракции и дериватизации. Вторая часть посвящена клиническому применению метода ГХ-МС для диагностики различных форм эндогенного гиперкортизолизма, адренокортикального рака, врожденной дисфункции коры надпочечников, ферментативных нарушений метаболизма стероидов.

\section{ВВЕДЕНИЕ}

Методы хроматографического анализа получили широкое применение в различных отраслях знаний, в том числе и медицине. Они открывают широкие перспективы и большие возможности для изучения процессов метаболизма, ключевых звеньев патогенеза, прогнозирования и диагностики заболеваний надпочечников [1, 2]. Развитие современных медицинских технологий расширило возможности ранней доклинической диагностики по выявлению больных с патологией коры надпочечников, как с опухолями, так и с неопухолевыми формами. Несмотря на достигнутые успехи в этой области, клиницисты сталкиваются с трудностями в оценке гормональной активности опухолей надпочечников, дифференциальной диагностики органического и функционального эндогенного гиперкортицизма. Осложнена диагностика заболеваний, протекающих с субклиническим течением: неклассических форм (НФ) врожденной дисфункции коры надпочечников (ВДКН) и автономной секреции кортизола (АСК). Диагностика на данном этапе заболевания требует изучения промежуточных продуктов адреналового стероидогенеза и их метаболитов. Важное значение приобретают методы жидкостной и газовой хроматомасс-спектрометрии, с помощью которых можно получать стероидные профили мочи (СПМ) и крови - наиболее ценные диагностические тесты заболеваний, связанных с нарушением синтеза и метаболизма стероидных гормонов [2-4]. На сегодняшний день подобная практика уверенно входит в процесс лабораторной диагностики за рубежом и все прочнее укрепляет свои позиции в России. Более того, группа исследователей из университета Манчестера, проанализировав данные из различных источников по определению стероидов методами иммуноанализа и хромато-массспектрометрии, пришла к выводу, что в скором времени последние вытеснят иммуноферментные методы не только в исследовательских, но и клинико-биохимических лабораториях [5]. 
За последние годы появились работы в области дифференциальной диагностики адренокортикальной аденомы (АКА) и адренокортикального рака (АКР) [6-9], ранних признаков злокачественности инциденталом надпочечников (ИН) [10, 11], различных форм эндогенного гиперкортицизма [12, 13], первичного гиперальдостеронизма [14], дефектов адреналового стероидогенеза $[15,16]$. Среди хроматографических методов для этих целей применяют высокоэффективную жидкостную хроматографию (ВэЖХ) [17, 18], газовую и жидкостную хромато-масс-спектрометрию (ЖХ-МС) [6-14]. Наиболее информативным для диагностики заболеваний надпочечников является исследование СПМ методом ГХ-МС, позволяющее за счет высокой разрешающей способности определять более 40 показателей (андрогенов, глюкокортикоидов, минералокортикоидов и их предшественников) за один цикл [4] (табл. 1).

\section{ПРОБОПОДГОТОВКА}

Физиологические жидкости, такие как кровь и моча, представляют собой сложные биологические матрицы, содержащие помимо аналитов другие биохимические компоненты, соли или примеси, мешающие определению целевых веществ. "Матричные эффекты" приводят к снижению чувствительности при детектировании стероидов в биологической матрице по сравнению со стандартными образцами в чистом растворителе. Кроме того, стероидытруднолетучие соединения, которые разлагаются при высоких температурах, необходимых для проведения газохроматографического анализа. Все эти проблемы должна решить пробоподготовка образцов мочи перед ГХ-анализом стероидных гормонов (стадии экстракции и дериватизации).

Гидролиз. Стероидные гормоны в моче находятся в форме глюкуронидных, сульфатных и глютатионовых конъюгатов. Перед проведением ГХ-МС-анализа конъюгаты расщепляют путем ферментативного гидролиза с $\beta$-глюкуронидазой или смесью $\beta$-глюкуронидазы с сульфатазой /арилсульфатазой, полученными, в основном, из Escherichia coli (E. coli) и Helix pomatia. Последний фермент получил более широкое применение, поскольку, в отличие от E. Coli, он способен деконъюгировать не только глюкуронидные формы, но и сульфатные [19]. Время и температура, необходимые для расщепления конъюгатов, приводятся разные, от 2 ч при $55^{\circ} \mathrm{C}$ до 12-72 ч при "классической" температуре $37^{\circ} \mathrm{C}$ [4, 20-22]. В то же время Helix pomatia имеет существенный недостаток: в процессе гидролиза наблюдаются различные явления трансформации стероидов, что приводит к узкому линейному диапазону определения некоторых стероидных гормонов [23]. Добавка аскорбата натрия в ферментативный раствор предотвращает потери нестабильных соединений [3].

Экстракция. Для извлечения стероидов из биологических жидкостей применяют, в основном, два варианта экстракции - твердофазный (ТФЭ) $[6,12,16,21$, 24] и жидкостно-жидкостный (ЖЖЭ) [4, 8-10, 14, 18, 24, 25]. Возможна комбинация этих двух вариантов экстракции [24]. Для ЖЖЭ применяют дихлорметан [18, 25], трихлорметан [8], этилацетат [9, 20]. Органический слой упаривают досуха током азота или воздуха. За последние годы появилось много вариантов ЖЖЭ стероидов. К ним относятся жидкофазная микроэкстракция с использованием мембраны с полым волокном [26], ион-парная экстракция [27], дисперсионная жидкостно-жидкостная микроэкстракция ионной жидкостью [17]. Материалом для ТФЭ (или сорбционного концентрирования) служит силикагель с привитыми фазами [6, 12, 16, 21], полимерные сорбенты [28] и молекулярно-импринтированные полимеры [29]. Биологическую жидкость пропускают через картридж с сорбентом с последующим промыванием (водой и / или органическими растворителями) и элюированием стероидов этанолом [16], метанолом [21] или смесью растворителей [24]. К недостаткам неполярных сорбентов на основе силикагеля Sep-Pak C18 и полярных $\mathrm{NH}_{2}$-сорбентов с амино-пропильными группами можно отнести их зависимость от рН, что неизбежно приводит к частичной потере образца. Поэтому для ТФЭ-стероидов предпочтительны сорбенты на основе сополимеров стирола и дивинилбензола (XAD), дивинилбензола и N-винилпирролидона (OasisHLB) [30]. На сегодняшний день нет однозначного ответа на вопрос, какой вариант экстракции стероидов является оптимальным. Важно то, что правильно подобранная стратегия пробоподготовки позволяет избежать ошибки всего анализа.

Дериватизация. В случае термически неустойчивых кортикостероидов необходимо получение более летучих и менее полярных производных. Наиболее широкое распространение для этих целей получила реакция силилирования - превращение гидроксильных групп в триметилсилильные или трет-бутилдиметилсилильные эфиры. В качестве дериватизирующих агентов в чистом виде или в виде смесей используют N-(триметилсилил)-имидазол (TSIM) [4], N, O-бис(триметилсилил)-трифторацетамид (BSTFA) [21], $\mathrm{N}$-метил-N-(триметилсилил)-трифторацетамид (MSTFA) [31]. Важно отметить, что в процессе дериватизации происходит енолизация кетогрупп с образованием триметилсилиловых эфиров енольных форм. Спектры енол-TMS производных менее информативны для анализа структуры стероидов из-за меньшей фрагментации. В клинической практике перед силилированием проводят превращение кетогрупп в метоксимные (двойная дериватизация) [32]. Метоксиамин гидрохлорид, растворенный в пиридине, дает полную реакцию при $60{ }^{\circ} \mathrm{C}$ в течение 1-1,5 ч за исключением карбонильной группы в С-11 положении, которая не образует оксима. Продолжительность реакции силилирования изменяется от 20 мин при $70{ }^{\circ} \mathrm{C}$ до 8-12 ч при $100^{\circ} \mathrm{C}[4,6]$. 
Таблица 1. Основные стероиды мочи и их метаболиты, определяемые методом газовой хромато-масс-спектрометрии

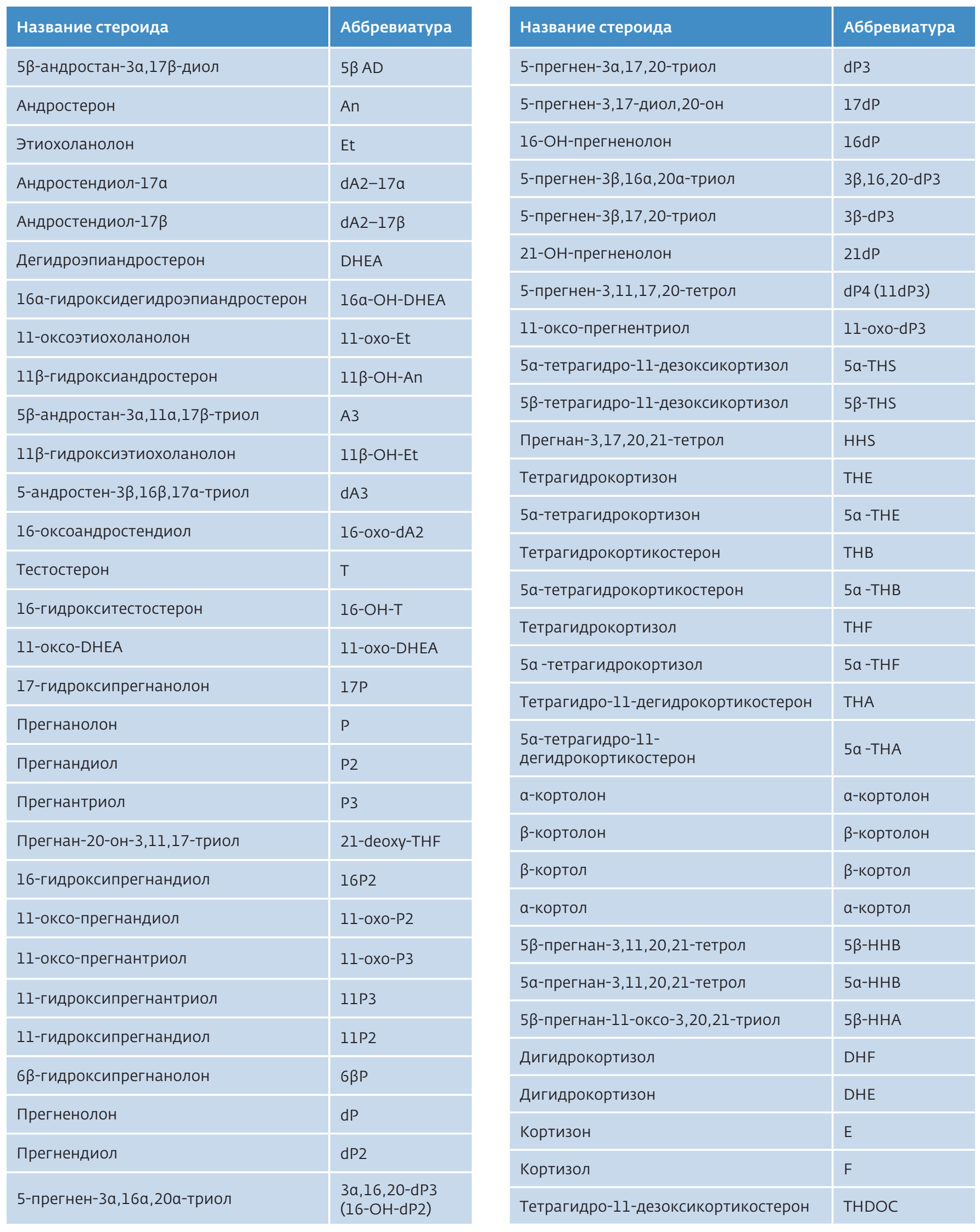




\section{ГАЗОХРОМАТОГРАФИЧЕСКИЙ АНАЛИЗ}

Л. И. Великанова и др. (2016 год) исследовали СПМ на газовом хромато-масс-спектрометре SHIMADZU GCMS - QP2010 Ultra. Авторы использовали капиллярную колонку Rtx-5MS (30 м × 0,25 мм × 0,25 мкм; Restek, США), объем вводимой пробы-3 мкл; газ-носитель - гелий; температурный градиент в диапазоне: 50-270 C. Детектируемая масса: 100-900 m/ z, время сканирования - 0,3 с [4]. Другие исследователи использовали ГХ-МС-системы Agilent 5973 [7, 34]; HР 5973 (Hewlett-Packard, Wilmington, Германия) [15]; газовый хроматограф Hewlett Packard 5890 с масс-спектрометром Finnigan MAT SSQ 710 [12]; газовый хроматограф Agilent 7890A с массселективным детектором Agilent 5975C (Agilent Technologies, SantaClara, CA, USA) [16, 31]; газовый хроматограф с массселективным детектором Agilent 6890N-5973I (HewlettPackard, PaloAlto, США) [26]. Для разделения стероидов применяли капиллярные колонки DB1 (15 м, J and W Scientific, Folsom, США) [15], Agilent HP-5MS (30 м × 0,25 мм × 0,25 мКм; Agilent Technologies, Santa Clara, СШA) [16], Agilent HP1-Ultra $(17 \mathrm{M} \times 200 \mathrm{MKM} \times 0,11 \mathrm{MKM}$; Agilent Technologies, CШA) [31], DB-5MS (30 M ×0,25 мM $\times 0,25$ mKM, Agilent Technologies) [26].

Возможность определения широкого спектра стероидных гормонов методом ГХ-МС увеличивает точность диагностики "гормонально-неактивных" аденом (ГНА) коры надпочечников, адренокортикального рака на дооперационном этапе, различных форм врожденной дисфункции коры надпочечников (ВДКН) [6-11, 15, 16, 32].

"Гормонально-неактивные" аденомы коры надпочечников. Ряд ученых провели анализ 28 образцов пациентов с ГНА, и с помощью иерархического кластерного анализа и метода главных компонент выявили потенциальные биомаркеры (а-кортол, THE, THF, 5a-THF и Et), которые могут указывать на возрастающую активность ИН и возможное субклиническое течение заболевания [21]. На основании сочетания методов иммуноанализа и ГХ-МС Л.И. Великанова и др. (2015 год) диагностировали ГНА коры надпочечников у $8,6 \%$ пациентов с ИН, АСК - у 22,9\%, ранние признаки злокачественности опухоли коры надпочечников установлены у 7,6\%, недостаточность ферментов 21-гидроксилазы и 11ß-гидроксилазы - у 16,2\% [33]. Методом ГХ-МС были получены дополнительные признаки АСК: снижение экскреции с мочой DHEA и его метаболитов, увеличение экскреции с мочой 5 $\beta$-ТНF и 5 $\beta$-ТНВ (признаки снижения активности 5а-редуктазы), соотношений THF/THЕ и THB/THА (признаки снижения активности 11ß-гидроксистероиддегидрогеназы 2 типа) [33].

Диагностика адренокортикального рака. Метод ГХ-МС приобретает особую роль в дифференциальной диагностике АКА и АКР. В отличие от альдостерон- или кортизолпродуцирующих аденом, стероидный биосинтез при АКР является многонаправленным и характеризуется большим количеством метаболитов предшественников андрогенов, кортизола и альдостерона. Контроль содержания биомаркеров АКР, которые не определяются методами иммуноанализа и ЖХ-МС, позволяет диагностировать АКР на ранней стадии до проявления клинической прогрессии заболевания [10]. В 2011 году было проведено крупное исследование по изучению методом ГХ-МС экскреции с мочой 32 стероидов у 102 пациентов с АКА и 45 - с АКР [7]. Было выделено 9 наиболее информативных стероидов. Самым дискриминационным стероидом был признан THS, уровень которого был значительно повышен у больных АКР.

В 2015 году группа исследователей из Голландии представила свои данные о независимой когорте пациентов с АКР и АКА. Из 22 проанализированных метаболитов стероидов 15 были выделены в качестве отдельных стероидных маркеров с чувствительностью более 90\% при обнаружении АКР, а уровень экскреции с мочой THS снова оказался наиболее значимым критерием [34]. Другие информативные маркеры, идентифицированные в этом исследовании, включали Р2, P3, Et, An, THF и THE, что согласуется с данными, полученными ранее [7].

Л.И. Великанова и др. (2016 год) исследовали СПМ 52 пациентов с ГНА и 31 больного АКР методами ГХ-МС и ВЭЖХ [8]. Методом ГХ-МС было исследовано 66 стероидов. Выделено четыре типа СПМ, характерных для АКР, у больных синдромом Кушинга (СК) и у пациентов без АСК, которые различались по глюкокортикоидной и андрогенной функции коры надпочечников. Получены 22 биомаркера AKP: DHEA и его метаболиты (16-OH-DHEA, dA2-17ß, dA3, 16-oxo-dA2), метаболиты андростендиона (Et, $11 \beta-\mathrm{OH}-\mathrm{Et})$, THS, метаболиты прогестерона и 17-ОН-прогестерона (17P, 6ßР, Р2, Р3, 11-охо-Р3), метаболиты прегненолона и 17-ОН-прегненолона (dP2, dP3-3a, 3a, 16, 20-dP3). У больных АКР были определены "неклассические" 5-еnе-прегнены, не обнаруженные у здоровых лиц и у пациентов с новообразованиями надпочеч-

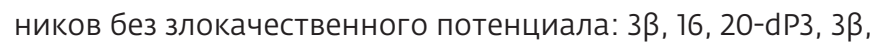
17, 20-dP3, 16dP, 21dP, 11dP3, 21dP2. Основными биомаркерами AKР оказались DHEA (>1500 мкг/24 ч) и THS (>1000 мкг/24 ч), которые в сочетании с определением "неклассических" 5-еnе-прегненов позволили получить 100\%-ную чувствительность и специфичность дифференциальной диагностики АКА и АКР [8].

Ранние признаки малигнизации надпочечников были установлены при исследовании СПМ методом ГХ-МС у больных с аденомой коры надпочечников и злокачественным потенциалом (3П) 1-3 балла по шкале L. M.Weiss, а также у пациентов с феохромоцитомой (ФХ) с агрессивным течением опухоли (больше четырех баллов по шкале PASS) [10]. Для этих больных было характерно увеличение экскреции с мочой андрогенов (Et, DHEA и его метаболитов), метаболитов их предшественников (P2, Р3, dP3, 16-OH-dP2) и THS. У данных пациентов, как и у больных АКР, были обнаружены «неклассические» 5-enе-прегнены. У больных СК и зП 1-3 балла по шкале L. M. Weiss 100\%-ная чувствительность 
и специфичность были получены для экскреции с мочой THS более 500 мкг / 24 ч [11].

Врожденные дисфункции коры надпочечников. В настоящий момент стероидное профилирование играет ключевую роль в изучении наследственных нарушений синтеза, метаболизма и оказываемого действия стероидных гормонов. Генетически выявленные биосинтетические дефекты, встречающиеся, в основном, в детском возрасте, обусловлены наличием ферментативной недостаточности надпочечников и/ или избыточной продукцией дефектных субстратов энзима. Наиболее частая причина ферментативной недостаточности коры надпочечников у младенцев - ВДКН, которая в 95\% случаев связана с дефектом 21-гидроксилазы (21-Г), 5\% приходятся на недостаточность 11ß-гидроксилазы (11 -Г), 17а-гидроксилазы

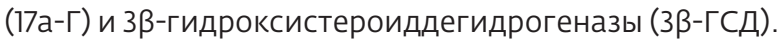

В 2002 году М. P. Caulfield и др. изучили профили 34 стероидов больных ВДКН, обусловленной в 31 случае дефектом 21-Г, два случая приходилось на дефект 11 $\beta-Г$, двое пациентов имели недостаточности 17a-Г или 3ß-ГСД [15]. Определены референсные значения для 3 $\beta$-гидрокси-5-ene-стероидов и метаболитов DHEA и прегненолона, а также диагностические соотношения прекурсор / метаболит, отражающие активность соответствующих ферментов. Полученные результаты позволили дифференцировать все четыре вида недостаточности. В другом исследовании СПМ методом ГХ-МС авторы получили одновременно признаки НФ ВДКН вследствие дефектов 21-Г и 11ß-Г [35].

В работе Н. В. Ворохобиной и др. (2016 год) были исследованы СПМ методом ГХ-МС у 54 женщин с гиперандрогенией (ГА) [36]. НФ ВДКН вследствие дефекта 21-Г установлена у $14,8 \%$ пациентов с ГА, синдром поликистозных яичников (СПКЯ) - у 33,3\%. У больных НФ ВДКН с дефектом 21-Г авторы получили увеличение экскреции с мочой 17P, P3, 11-oxo-P3, DHEA, 16-oxo-dA2, 21-deoxy-THF и определили "неклассические» 5-ene-прегнены. Для диагностики НФ ВДКН с дефектом 21-Г были получены пороговые значения соотношений: (THF +5a-THF + THE) /11-охо-P3<30 и (THF+5a-THF+THE)/P3<3. Четыре признака увеличения активности 5а-редуктазы были получены у больных СПКЯ, два признака - у пациентов с НФ ВДКН вследствие дефекта 21-Г.

В 2016 году исследователи провели анализ суточной мочи 87 детей с ожирением [37]. Данные были Z-преобразованы на основании половой и возрастной принадлежности. Примененный метод главных компонент позволил выделить пять кластеров. В первом не было обнаружено значительных отличий от референсной группы, тогда как для образцов из второго кластера было выявлено неспецифическое увеличение С19 и С21 стероидов. В третьем кластере были обнаружены различия для Р3, 17Р, $\beta$-кортола, 11-ОН-An, 11-охо-Р3, THS, увеличение экскреции которых с мочой могло свидетельствовать о наличии дефекта 21-Г.
При сравнении Z-преобразованных величин значительные различия были обнаружены для 17ß-dA2, 17ß-dA3, P2, F, THE, THF, $\beta$-кортолона, а-кортола, 6ß-OH-F, DHF, 11-охо-An, 11-OHEt, THB в четвертом кластере. Для пациентов из четвертой группы были характерны заметное повышение уровня С21стероидов и дисбаланс в 11 -гидроксистероиддегидрогеназн ой системе. Больные из пятого кластера оказались склонны к повышенной секреции метаболитов DHEA и 17-гидроксипрегненолона, что могло указывать на недостаточность зß-гидроксистероиддегидрогеназы. Авторы уверены, что стероидное профилирование позволяет уточнить понятие "ожирение", а применение кластерного подхода будет полезным в развитии персонализированной медицины.

\section{ЗАКЛЮЧЕНИЕ}

Хроматографические методы анализа в сочетании с масс-спектрометрическим детектированием широко применяются в диагностике различных заболеваний за рубежом. За последнее десятилетие наблюдается тенденция к возрастанию интереса к проведению подобных исследований и в России. Сочетание классических методов иммуноанализа с исследованием СПМ методом ГХ-МС позволило внести значительный вклад в дифференциальную диагностику инциденталом надпочечников, различных форм эндогенного гиперкортицизма и нарушений адреналового стероидогенеза, в диагностику адренокортикального рака.

\section{ЛИТЕРАТУРА}

1. Storbeck K.H., Schiffer L., Baranowski E. S., Chortis V., Prete A., Barnard L., et al. Steroid metabolome analysis in disorders of adrenal steroid biosynthesis and metabolism // Endocrine Reviews, https://doi. org/10.1210/er.2018-00262.

2. Keevil B. G. LC-MS/MS analysis of steroids in the clinical laboratory // Clin. Biochem. 2016. V. 49. I. 13-14. P. 989-997.

3. Taylor N. F. Urinary steroid profiling // Methods Mol. Biol. 2013. V. 1065. P. 259-276.

4. Великанова Л.И., Стрельникова Е.Г., Объедкова Е. В., Кривохижина Н.С., Шафигуллина 3.Р., Григорян К. и др. Получение стероидных профилей мочи больных с инциденталомой надпочечников методом газовой хромато-масс-спектрометрии // Журн. анал. химии. 2016. Т. 71. № 7. С. 775-781.

5. Taylor A. E., Keevil B., Huhtaniemi I. T. Mass spectrometry and immunoassay: how to measure steroid hormones today and tomorrow // Eur. J. Endocrinol. 2015 V. 173. I. 2. P. 1-12.

6. Tiu S. C., Chan A. O., Taylor N. F., Lee C. Y., Loung P. Y., Choi C. H., et al. Use of urinary steroid profiling for diagnosing and monitoring adrenocortical tumors // Hong Kong Med. J. 2009. V. 15. P. 463470.

7. Arlt W., Biehl M., Taylor A. E., Hahner S., Libe R., Hughes B. A., et al. Urine steroid metabolomics as a biomarker tool for detecting malignancy in adrenal tumors // J. Clin. Endocrin. Metab. 2011. V. 96. P. 3775-3784.

8. Velikanova L. I., Shafigullina Z. R., Lisitsin A. A., Vorokhobina N. V., Grigoryan K., Kukhianidze E. A., et al. Different types of urinary steroid profiling obtained by high-performance liquid chromatography and gas chromatography mass spectrometry in patients with adrenocortical carcinoma // Horm. Cancer. 2016. V.7. I. 5-6. P. 327-335.

9. Taylor D. R., Ghataore L., Couchman L., Vincent R. P., Whitelaw B., Lewis D., et al. A 13-steroid serum panel based on LC-MS/MS: Use in detection of adrenocortical carcinoma // Clin. Chem. 2017. V. 63. I. 12. P. 1836-1846. 
10. Shafigullina Z. R., Velikanova L. I., Vorokhobina N. V., Shustov S. B., Lisitsin A. A., Malevanaia E. V., et.al. Urinary steroid profiling by gas chromatography - mass spectrometry: Early features of malignancy in patients with adrenal incidentalomas // Steroids. 2018. V. 135. P. 31-35.

11. Великанова Л.И., Шафигуллина 3.Р., Ворохобина Н.В., Малеваная Е.В. Определение ранних признаков злокачествен ности новообразований надпочечников у больных синдромом Кушинга в результате изучения метаболомики стероидов мочи методом газовой хромато-масс-спектрометрии // Бюллетень экспериментальной биологии и медицины. 2019. Т. 167. № 5. C. 621-626.

12. Kotlowska A., Puzyn T., Sworczak K., Stepnowski P., Szefer P. Metabolomic biomarkers in urine of Cushing's syndrome patients // Int. J. Mol. Sci. 2017. V. 18. Р. 294-308.

13. Шафигуллина 3.Р., Великанова Л.И., Ворохобина Н.В., Лисицын А.А., Кухианидзе Е.А., Стрельникова Е.Г. и др. Диагностическое значение стероидных профилей биологических жидкостей у больных синдромом Иценко - Кушинга // Проблемы эндокринологии. 2015. Т. 61. № 4. С. 4-8.

14. Eisenhofer G., Dekkers T., Peitzsch M., Dietz A.S., Bidlingmaier M., Treitl M., et al. Mass spectrometry-based adrenal and peripheral venous steroid profiling for subtyping primary aldosteronism // Clin. Chem. 2016. V. 62. I. 3. P. 514-524.

15. Caulfield M. P., Lynn T., Gottschalk M. E., Jones K. L., Taylor N. F., Malunowicz E.M., et al. The diagnosis of congenital adrenal hyperplasia in the newborn by gas chromatography/mass spectrometry analysis of random urine specimens // J. Clin. Endocrinol. Metab. 2002. V. 87. P. 3682-3690.

16. Tran M.T.C., Tran N.A.T., Nguyen P.M., Vu C.D., Tran M.D., Ngo D. N., et al. 11ß-Hydroxylase deficiency detected by urine steroid metabolome profiling using gas chromatography-mass spectrometry // Clin. Mass. Spectr. 2018. V. 7. P. 1-5.

17. Abujaber F., Corps R. Al., Ríos Ā., Guzmán B. F.J., Rodríguez M.-D.R.C. Ionic liquid dispersive liquid-liquid microextraction combined with LC-UV-Vis for the fast and simultaneous determination of cortisone and cortisol in human saliva samples // J. Pharm. Biomed. Anal. 2018. V. 165. P. 141-146.

18. Obedkova E. V., Kartsova L. A., Kirsanov D. O., Velikanova L. I., Legin A. V. Generation of characteristic profiles of steroid hormones by reversed-phase HPLC // J. Anal. Chem.2014. V. 69. I. 2. P. 200-204.

19. Graef V., Furuya E., Nishikaze O. Hydrolysis of steroid glucuronides with beta-glucuronidase preparations from bovine liver, Helix pomatia, and E. coli // Clin. Chem. 1977. V. 23. I. 3. P. 532-535.

20. Hines J. M., Bancos I., Bancos C., Singh R. D., Avula A. V., Young W. F., et al. High-Resolution, Accurate-mass (HRAM) mass spectrometry urine steroid profiling in the diagnosis of adrenal disorders // Clin. Chem. 2017. V. 63. I. 11. P. 1824-1835.

21. Kotlowska A., Sworczak K., Stepnowskic P. Urine metabolomics analysis for adrenal incidentaloma activity detection and biomarker discovery // J. Chrom. B. 2011. V. 879. P. 359-363.

22. Christakoudi S., Cowan D. A., Christakudis G., Taylor N. F. 21-Hydroxylase deficiency in the neonate - trends in steroid anabolism and catabolism during the first weeks of life // J. Steroid Biochem. Mol. Biol. 2013. V. 138.P. 334-347.

23. Moon J.Y., Ha Y.W., Moon M. H., Chung B. C., Choi M. H. Systematic error in gas chromatography-mass spectrometry-based quantification of hydrolyzed urinary steroids // Cancer Epidemiol. Biomarkers Prev. 2010. V. 19. I. 2. P. 388-397.

24. Doué M., West C., Bichona E., Bizec B. L., Lesellier E. Supercritical fluid chromatography applied to the highly selective isolation of urinary steroid hormones prior to GC/MS analysis // J. Chromatogr. B. 2018. V. 1086. P. 97-104.

25. Moon J.Y., Lee H.S., Kim J.H., Lee J.H., Choi M.H. Supported liquid extraction coupled to gas chromatography-selective mass spectrometric scan modes for serum steroid profiling // Anal. Chim. Acta. 2018. V. 1037. P. 281-292.

26. Liu W., Zhang L., Fan L., Lin Z., Cai Y., Wei Z., et al. An improved hollow fiber solvent-stir bar microextraction for the preconcentration of anabolic steroids in biological matrix with determination by gas chromatography-mass spectrometry // J. Chromatogr. A. 2012. V. 1233. P. 1-7.

27. Cawley A. T., Kazlauskas R., Trout G. J., George A. V. Determination of urinary steroid sulfate metabolites using ion paired extraction // J. Chromatogr. B Anal. Technol. Biomed. Life Sci. 2005. V. 825. P. 1-10.

28. Kole P. L., Venkatesh G., Kotecha J., Sheshala R. Recent advances in sample preparation techniques for effective bioanalytical methods // Biomed. Chromatogr. 2011. V. 25. P. 199-217.

29. Gadzala-Kopciuch R., Ricanyova J., Buszewski B. Isolation and detection of steroids from human urine by molecularly imprinted solid-phase extraction and liquid chromatography // J. Chromatogr. B. 2009. V. 877. P. 1177-1184.

30. Cho Y. D., Choi M. H. Alternative sample preparation techniques in gas chromatographic mass spectrometric analysis of urinary androgenic steroids // Bull. Kor. Chem. Soc. 2006. V. 27. I. 9.P. 1315-1322.

31. Teubel J., Wüst B., Schipke C. G., Parr M. K. Methods in endogenous steroid profiling - A comparison of gas chromatography mass spectrometry (GC-MS) with supercritical fluid chromatography tandem mass spectrometry (SFC-MS/MS) // J. Chromatog. A. 2018. V. 1554. P. 101-116.

32. Honour J.W., Conway E., Hodkinson R., Lam F. The evolution of methods for urinary steroid metabolomics in clinical investigations particularly in childhood // J. Steroid Biochem. Mol. Biol. 2018. V. 181. Р. 28-51.

33. Великанова Л.И., Шафигуллина 3.Р., Ворохобина Н.В., Григорян К., Лисицын А. А., Объедкова Е. В. Дифференциальная диагностика инциденталом коры надпочечников различными лабораторными технологиями // Вестник Северо-Западного гос. мед. ун-та. 2015. Т. 7. № 4. С. 52-58.

34. Kerkhofs T. M., Kerstens M. N., Kema I. P., Willems T. P., Haak H. R. Diagnostic value of urinary steroid profiling in the evaluation of adrenal tumors // Horm. Cancer. 2015. V. 6. I. 4.P. 168-175.

35. Ворохобина Н.В., Великанова Л.И., Шафигуллина 3.Р., Шустов С. Б., Стрельникова Е. Г. Бессонова Е. А. Способ одновременной диагностики неклассических форм врожденной дисфункции коры надпочечников с недостаточностью 21-гидроксилазы и 11ß-гидроксилазы / Пат. 2649801 Российская Федерация. Заяв.ипатентообл. ФГБОУ ВО СЗГМУ им. И. И. Мечникова. № 2016152672, заявл. 29.12.2016, опубл. 04.04.2018, Бюл. № $10.18 \mathrm{C}$.

36. Ворохобина Н.В., Татаринова М.В., Великанова Л.И., Серебрякова И. П., Малеваная Е. В., Галахова Р. К. Особенности метаболизма стероидных гормонов у женщин репродуктивного возраста с различными формами гиперандрогении // Вестник Северо-Западного гос. мед.ун-та. 2016. Т. 8. № 3. С. 42-49.

37. Gawlik A., Shmoish M., Hartmann M.F., Malecka-Tendera E. Wudy S. A., Hochberg Z. Steroid metabolomic disease signature of non syndromic childhood obesity // J. Clin. Endocrinol. Metab. 2016. V. 101. I. 11. P. 4329-4337.

\section{REFERENCES}

1. Storbeck K.H., Schiffer L., Baranowski E.S., Chortis V., Prete A., Barnard L., et al. Steroid metabolome analysis in disorders of adrenal steroid biosynthesis and metabolism // Endocrine Reviews, https://doi. org/10.1210/er.2018-00262.

2. Keevil B. G. LC-MS/MS analysis of steroids in the clinical laboratory // Clin. Biochem. 2016. V. 49. I. 13-14. P. 989-997.

3. Taylor N. F. Urinary steroid profiling // Methods Mol. Biol. 2013. V. 1065. P. 259-276.

4. Velikanova L.I., Strel'nikova E.G., Obedkova E.V., Krivokhizhina N.S., Shafigullina Z. R., Grigoryan K., et al. Generation of urinary steroid profiles in patients with adrenal incidentaloma using gas chromatography - mass spectrometry. Zhurnal analiticheskoj himii - Journal of Analytical Chemistry, 2016, vol. 71, no. 7, pp. 775781.

5. Taylor A. E., Keevil B., Huhtaniemi I.T. Mass spectrometry and immunoassay: how to measure steroid hormones today and tomorrow // Eur. J. Endocrinol. 2015. V. 173. I. 2. P. 1-12. 
6. Tiu S. C., Chan A. O., Taylor N.F., Lee C.Y., Loung P.Y. Choi C. H., et al. Use of urinary steroid profiling for diagnosing and monitoring adrenocortical tumors // Hong Kong Med. J. 2009. V. 15. P. 463-470.

7. Arlt W., Biehl M., Taylor A. E., Hahner S., Libe R., Hughes B. A., et al. Urine steroid metabolomics as a biomarker tool for detecting malignancy in adrenal tumors // J. Clin. Endocrin. Metab. 2011. V. 96. P. 3775-3784.

8. Velikanova L. I., Shafigullina Z. R., Lisitsin A. A., Vorokhobina N. V., Grigoryan K., Kukhianidze E.A., et al. Different types of urinary steroid profiling obtained by high-performance liquid chromatography and gas chromatography mass spectrometry in patients with adrenocortical carcinoma // Horm. Cancer. 2016. V. 7. I. 5-6. P. $327-335$

9. Taylor D. R., Ghataore L., Couchman L., Vincent R. P., Whitelaw B., Lewis D., et al. A 13-steroid serum panel based on LC-MS/MS: Use in detection of adrenocortical carcinoma // Clin. Chem. 2017. V. 63. I. 12. P. 1836-1846.

10. Shafigullina Z. R., Velikanova L. I., Vorokhobina N. V., Shustov S. B., Lisitsin A. A., Malevanaia E. V., et.al. Urinary steroid profiling by gas chromatography - mass spectrometry: Early features of malignancy in patients with adrenal incidentalomas // Steroids. 2018. V. 135 P. 31-35.

11. Velikanova L.I., Shafigullina Z.R., Vorohobina N.V., Malevanaia E. V. Gas chromatography-mass spectrometry analysis of urinary steroid metabolomics for detection of early signs of adrenal neoplasm malignancy in patients with Cushing's syndrome. Bjulleten' jeksperimental"noj biologii i mediciny - Bulletin of Experimental Biology and Medicine, 2019, vol. 167, no. 5, pp. 621-626.

12. Kotlowska A., Puzyn T., Sworczak K., Stepnowski P., Szefer P. Metabolomic biomarkers in urine of Cushing's syndrome patients // Int. J. Mol. Sci. 2017. V. 18. P. 294-308.

13. Shafigullina Z. R., Velikanova L. I., Vorohobina N. V., Lisicyn A. A., Kuhianidze E. A., Strel'nikova E.G. et al. The diagnostical importance of steroid profiles of biological fluids of patients with Cushing's syndrome. Problemy jendokrinologii - Problems of endocrinology, 2015, vol. 61, no. 4, pp. C. 4-8.

14. Eisenhofer G., Dekkers T., Peitzsch M., Dietz A.S., Bidlingmaier M., Treitl M., et al. Mass spectrometry-based adrenal and peripheral venous steroid profiling for subtyping primary aldosteronism // Clin. Chem. 2016. V. 62. I. 3. P. 514-524.

15. Caulfield M.P., Lynn T., Gottschalk M. E., Jones K. L., Taylor N. F., Malunowicz E.M., et al. The diagnosis of congenital adrenal hyperplasia in the newborn by gas chromatography/mass spectrometry analysis of random urine specimens // J. Clin. Endocrinol. Metab. 2002. V. 87. P. 3682-3690.

16. Tran M.T.C., Tran N. A. T., Nguyen P. M., Vu C. D., Tran M.D. Ngo D. N., et al. 11ß-Hydroxylase deficiency detected by urine steroid metabolome profiling using gas chromatography-mass spectrometry // Clin. Mass. Spectr. 2018. V. 7. P. 1-5.

17. Abujaber F., Corps R.Al., Ríos Ā., Guzmán B.F.J., Rodríguez M.-D.R.C. Ionic liquid dispersive liquid-liquid microextraction combined with LC-UV-Vis for the fast and simultaneous determination of cortisone and cortisol in human saliva samples // J. Pharm. Biomed. Anal. 2018. V. 165. P. 141-146.

18. Obedkova E. V., Kartsova L. A., Kirsanov D. O., Velikanova L. I., Legin A. V. Generation of characteristic profiles of steroid hormones by reversed-phase HPLC // J. Anal. Chem. 2014. V. 69. I. 2. P. 200-204.

19. Graef V., Furuya E., Nishikaze O. Hydrolysis of steroid glucuronides with beta-glucuronidase preparations from bovine liver, Helix pomatia, and E. coli // Clin. Chem. 1977. V. 23. I. 3. P. 532-535.

20. Hines J. M., Bancos I., Bancos C., Singh R. D., Avula A. V., Young W. F., et al. High-Resolution, Accurate-mass (HRAM) mass spectrometry urine steroid profiling in the diagnosis of adrenal disorders // Clin. Chem. 2017. V. 63. I. 11. P. 1824-1835.

21. Kotlowska A., Sworczak K., Stepnowskic P. Urine metabolomics analysis for adrenal incidentaloma activity detection and biomarker discovery // J. Chrom. B. 2011. V. 879. P. 359-363.

22. Christakoudi S., Cowan D.A., Christakudis G., Taylor N.F. 21-Hydroxylase deficiency in the neonate - trends in steroid anabolism and catabolism during the first weeks of life // J. Steroid Biochem. Mol. Biol. 2013.V. 138.P. 334-347.

23. Moon J.Y., Ha Y.W., Moon M. H., Chung B. C., Choi M. H. Systematic error in gas chromatography-mass spectrometry-based quantification of hydrolyzed urinary steroids // Cancer Epidemiol. Biomarkers Prev. 2010. V. 19. I. 2. P. 388-397.

24. Doué M., West C., Bichona E., Bizec B. L., Lesellier E. Supercritical fluid chromatography applied to the highly selective isolation of urinary steroid hormones prior to GC/MS analysis // J. Chromatogr. B. 2018. V. 1086. P. 97-104.

25. Moon J.Y., Lee H.S., Kim J.H., Lee J.H., Choi M. H. Supported liquid extraction coupled to gas chromatography-selective mass spectrometric scan modes for serum steroid profiling // Anal. Chim. Acta. 2018. V. 1037. P. 281-292.

26. Liu W., Zhang L., Fan L., Lin Z., Cai Y., Wei Z., et al. An improved hollow fiber solvent-stir bar microextraction for the preconcentration of anabolic steroids in biological matrix with determination by gas chromatography-mass spectrometry // J. Chromatogr. A. 2012. V. 1233. P. 1-7.

27. Cawley A. T., Kazlauskas R., Trout G. J., George A. V. Determination of urinary steroid sulfate metabolites using ion paired extraction // J. Chromatogr. B Anal. Technol. Biomed. Life Sci. 2005. V. 825. P. 1-10.

28. Kole P. L., Venkatesh G., Kotecha J., Sheshala R. Recent advances in sample preparation techniques for effective bioanalytical methods // Biomed. Chromatogr. 2011. V. 25. P. 199-217.

29. Gadzala-Kopciuch R., Ricanyova J., Buszewski B. Isolation and detection of steroids from human urine by molecularly imprinted solid-phase extraction and liquid chromatography // J. Chromatogr. B. 2009. V. 877. P. 1177-1184.

30. Cho Y.D., Choi M. H. Alternative sample preparation techniques in gas chromatographic mass spectrometric analysis of urinary androgenic steroids // Bull. Kor. Chem. Soc. 2006. V. 27. I. 9. P. 1315-1322.

31. Teubel J., Wüst B., Schipke C. G., Parr M. K. Methods in endogenous steroid profiling - A comparison of gas chromatography mass spectrometry (GC-MS) with supercritical fluid chromatography tandem mass spectrometry (SFC-MS/MS) // J. Chromatog. A. 2018. V. 1554. P. 101-116.

32. Honour J.W., Conway E., Hodkinson R., Lam F. The evolution of methods for urinary steroid metabolomics in clinical investigations particularly in childhood // J. Steroid Biochem. Mol. Biol. 2018. V. 181. P. 28-51.

33. Velikanova L. I., Shafigullina Z. R., Vorokhobina N. V., Grigoryan K., Lisicyn A. A., Obedkova E. V. Differential diagnostics of adrenocortical incidentalomas with different laboratory technologies. Vestnik SeveroZapadnogo gosudarstvennogo medicinskogo universiteta - Herald of the Northwestern State Medical University named after I. I. Mechnikov, 2015, vol. 7, no. 4, pp. 52-58.

34. Kerkhofs T. M., Kerstens M. N., Kema I. P., Willems T. P., Haak H. R. Diagnostic value of urinary steroid profiling in the evaluation of adrenal tumors // Horm. Cancer. 2015. V. 6. I. 4. P. 168-175.

35. Vorokhobina N. V., Velikanova L. I., Shafigullina Z. R., Shustov S. B., Strel'nikova E.G., Bessonova E. A. The way of the simultaneous diagnosis of non-classical forms of congenital adrenal hyperplasia with a deficiency of 21-hydroxylase and 11ß-hydroxylase. Patent 2649801 Rossijskaja Federacija. Zajavitel' i patentoobladatel' FSBEI HE «NorthWestern State Medical University named after I. I. Mechnikov. No. 2016152672, zajavleno 29.12.2016, opublikovano 04.04.2018. Bjulleten' No. 10,18 p.

36. Vorokhobina N.V., Tatarinova M.V., Velikanova L.I., Serebryakova I.P., Malevanaia E. V., Galahova R. K. Features of steroid hormone metabolism in fertile age females with various forms of hyperandrogenism. Vestnik Severo-Zapadnogo gosudarstvennogo medicinskogo universiteta - Herald of the Northwestern State Medical University named after I. I. Mechnikov, 2016, vol. 8, no. 3, pp. 42-49.

37. Gawlik A., Shmoish M., Hartmann M. F., Malecka-Tendera E., Wudy S. A., Hochberg Z. Steroid metabolomic disease signature of non syndromic childhood obesity // J. Clin. Endocrinol. Metab. 2016. V. 101. I. 11. P. 4329-4337. 


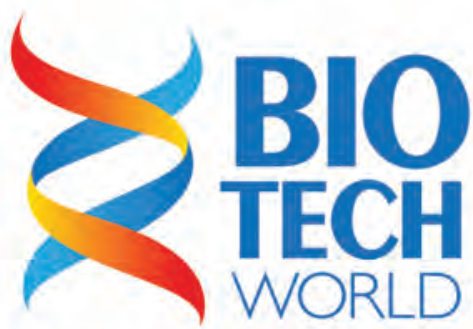

ХVIII МЕЖДУНАРОДНАЯ

СПЕЦИАЛИЗИРОВАННАЯ ВЫСТАВКА

БИОТЕХПРОМ \& АНАЛИТИКА

\section{РАЗДЕЛЫ ВЫСТАВКИ}

- Аналитика

- БИОФарма

- Функциональные продукты питания

- БИоЛаб

- БИОСофт

- БИОМед

- БИОАгро

- БИОВенчур, БИОФранчайзинг, Стартапы, Кластеры, Технопарки

- Промышленная биотехнология

- Лабораторная мебель БИОЛабДрайв

\section{БИОТЕХНОЛОГИЯ: \\ СОСТОЯНИЕ И \\ ПЕРСПЕКТИВЫ РАЗВИТИЯ}

\section{ОСНОВНЫЕ ПОТОКИ ФОРУМА}

- Фундаментальные вопросы биотехнологии

- Геномная инженерия

- Биотехнология и медицина

- Биофарма

- Биоинформатика

- Сельское хозяйство

- Life science

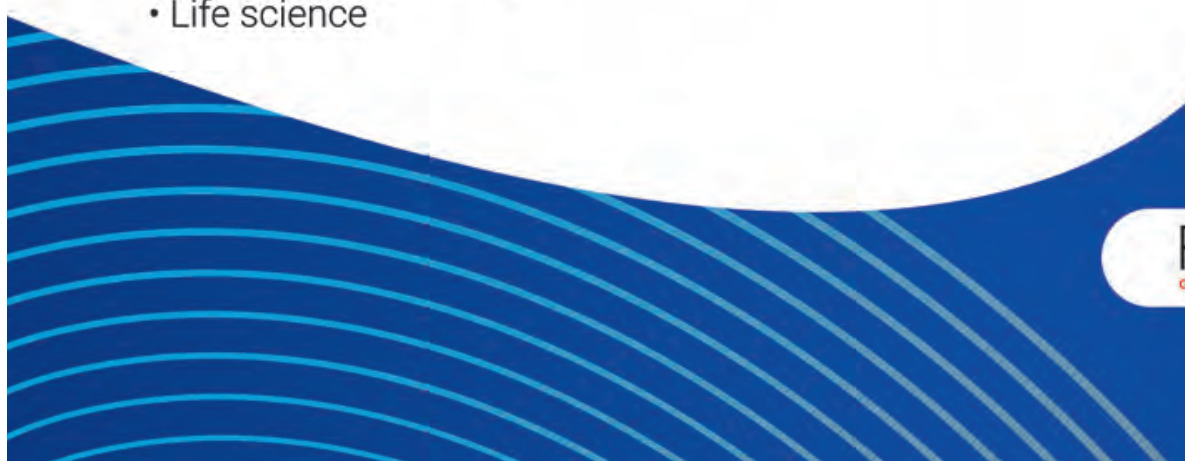

